\title{
Data Fusion Algorithms with State Delay and Missing Measurements
}

\author{
N Shivashankarappa*, Raol J. R**. \\ *(Professor, Dept. of Electronics and Communications Engineering, Acharya Institute of Technology (AIT) and \\ Jain University research scholar, Bangalore \\ **(Professor. Emeritus, MSRIT, Bangalore
}

\begin{abstract}
In wireless sensor networks and other engineering systems, there are situations wherein some delays occur in data transmission and some measurements might be randomly missing. This would cause inaccuracies in Kalman filter or its equivalent algorithms, when used for target tracking. In this paper four alternative algorithms are studied and the modifications to include the state delay and randomly missing measurements are provided. Especially:i) the gain fusion, $\mathrm{H}$-infinity a posteriori, H-infinity risk sensitive filter, and H-infinity global filtering algorithms are modified, and evaluated for sensor data fusion scenario using numerical simulations carried out in MATLAB; and ii) a nonlinear observer based on the continuous time data fusion filter is presented, and asymptotic convergence result is derived using Lyapunov energy functional; these two aspects are the novel contribution of this paper.
\end{abstract}

Keywords: Data fusion, delayed states, randomly missing measurements, gain fusion filter, H-infinity a posteriori filter, risk sensitive filter, $\mathrm{H}$-infinity global fusion filter, observer, Lyapunov function.

\section{INTRODUCTION}

The aspects of multi-sensor data fusion (MSDF) are very crucial for many civilian and military applications: like, target tracking, wireless sensor networks, WSN. The point is that one wants to ascertain the status and identity of the object under observation wherefore the measurements data are considered available from more than one sensor. Then,data fusion provides more information on the status of the object in some appropriate way [1]. However, in a communications channel a few or many measurements might be missing (from one or more sensors); here, it becomes necessary to evaluate the performance of these data processing/fusion algorithms in presence (and despite) of missing measurements; at certain times one might have only the random noises present in such channels. It is very important to study the problem of missing measurements in the filter/fusion processing cycle along with the state delays. This combined aspect has not gained much attention in the context of MSDF for target tracking, although certain aspects have been studied in certain special cases [2-10]. Refs.[2-4] considered some special apects: a) intermittent measurements, b) data missing in condition monitoring, and c) packet dropouts. A system with multiple sensor delay is considered [5], but the algoriuthm is very involved.

The effects of outliers and missing data are studied in [6], but the example is for simple time series case. The refs. $[7,8,10]$ deal with only missing observations, and the state delay is not considered. In
[9], the problem of system delay is studied. The measurements might be missing due to: a) a failure of one or more senor/s, and/or b) there could be a problem is a communication channel, and the received data might be only the channel noise. Thus, it is important to handle the situation of missing data in FFA in a formal way. Also, time delay is encountered in several real time systems, due to latency time of a few data-channels; and it is a key factor that could influence the overall system performance. If such aspects of system's state delay and misisng data are not handled suitableyin a target tracking and fusion filter, then the track might be lost.

In this paper, the state delay and radnomly missing measuremtns in filtering-cum-fusion algorithms (FFAs) are treated; and four existing filtering algorithms are modified to incldue thestate delay and randomly missing measurements: a) the gain fusion or the so called data sharing filter (GFA), b) H-infinity a posteriori filter (HIPF), c) H-infinity a posteriori risk sensitive filter (HIRF), and d) $\mathrm{H}$ infinity global fusion filter (HIGF). The performance metrics are evaluated using MATLAB based implementations of the four algorithms for randomly misisng measurements. Also, a nonlinear observer based on the continuous time data fusion filter is presented, and asymptotic convergence result for the observer error dynamics is derived using Lyapunov energy functional. The appropriate modifications of the four filtering-cum-data fusion algorithms, their evaluation and the stability analysis of the nonlinear 
observer error dynamics that is based on continuous time data fusion filterare the contributions of the present paper.

\section{FILTERING-CUM-FUSION ALGORIHTMS}

A nonlineardynamic system with the state delay is given as

$x(k+1)=f(x(k), x(k-1))+G w(k)(1)$

The measurement model for each sensor with missing data is given as

$$
z_{m}(k)=\beta_{m} H_{m} x(k)+v_{m}(k)
$$

In (2), $m=1,2$ is number of sensors. The process noise $\mathrm{w}($.$) , and measurement noise \mathrm{v}($.$) are assumed to be$ white Gaussian stochastic processes with the respective covariance matrices as $\mathrm{Q}$ and $\mathrm{R}$.

The quantity $\beta^{m}$ is a Bernoulli sequence which takes values 0 and 1 randomly; with $E\left\{\beta^{m}(k)=1\right\}=a^{m}(k)$ and $E\left\{\beta^{m}(k)=0\right\}=1-a^{m}(k)$, with 'a' as the percentage of measurements that arrive to the sensor fusion node; this also signifies that a few measurements are randomly missing. The constant $a$ is assumed to be known or pre-specified.

\subsection{Modified gain fusion filtering-cum-fusion algorithm (GFA)}

A gain fusion algorithm that is inherently a combination of the filtering and data fusion processingcapability [11] is modified here to incorporate the state delay and randomly missing measurements. It considers the local filters and one global fusion filter; in each scan, the local filters receive the measurements and after processing these, communicate the estimated states to the global filter. To start with, the time propagation of the global estimates is obtained as follows:

The state estimate is obtained in usual way as

$$
\tilde{x}(k+1)=F_{0} \hat{x}^{f}(k)+F_{1} \hat{x}^{f}(k-1)
$$

In (3), the Jacobians are given as

$$
F_{0}=\frac{\partial f(., .)}{\partial x} \text {, at } x=\hat{x}^{f}(k) ; F_{1}=\frac{\partial f(., .)}{\partial x} \text {, at } x=\hat{x}^{f}(k-1)
$$

The state-error covariance estimate is given as

$$
\tilde{P}^{f}(k+1)=F_{0} \hat{P}^{f}(k) F_{0}^{T}+F_{1} \hat{P}^{f}(k-1) F_{1}^{T}+G Q G^{T}
$$

Then, the local sensor level $(\mathrm{i}=1,2, \ldots)$ filters are reset as

$$
\begin{array}{r}
\tilde{x}_{m}(k+1)=\hat{x}^{f}(k+1) \\
\tilde{P}_{m}(k+1)=\hat{P}^{f}(k+1)
\end{array}
$$

The measurement updates of the local gains and the states is obtained as

$$
K_{m}=\left(\frac{1}{\gamma_{m}}\right) \beta_{m} \tilde{P}^{f}(k+1) H_{m}^{T}\left(\beta_{m}{ }^{2} H_{m} \tilde{P}^{f}(k+1) H_{m}^{T}+\frac{1}{\gamma_{m}} R_{m}\right)^{-1}
$$

$$
\hat{x}(k+1)=\tilde{x}^{f}(k+1)+K_{m}\left(z_{m}-\beta_{m} H_{m} \tilde{x}^{f}(k-1)\right)
$$

Then, the global fusion of ' $\mathrm{m}$ ' local estimates is obtained as

$$
\begin{aligned}
& \hat{x}(k+1)=\tilde{x}^{f}\left(\sum_{i}^{m}\left(\hat{x}^{i} k+1\right)-(m-1) \tilde{x}^{f}(k-1)\right)(9) \\
& \hat{P}^{f}(k+1)=\left[I-\sum_{i}^{m} \beta_{m} K^{k} H\right] P(k+1)\left[I-\sum_{i}^{m} \beta_{m} K^{i} H\right]^{T}+\sum_{i}^{m} K^{\dagger} R^{i} K^{t}
\end{aligned}
$$

\subsection{Modified H-infinity a posteriori filteringalgorithm (HIPF)}

This H-infinity filter is based on minimization of the H-infinity norm, and it is supposed to be a robust algorithm [12]. The basic filter is modified to suit the equations (1) and (2).The (covariance) state error Gramian (SEG) propagation is obtained for each sensor as

$$
\begin{aligned}
P_{m}(k+1)= & F_{0} P_{m}(k) F_{0}^{T}+F_{1} P_{m}(k-1) F_{1}^{T}+ \\
G Q G^{T}- & F_{0} P_{m}(k)\left[\beta_{m} H_{m}^{T} L_{m}^{T}\right] R_{m}^{-1}\left[\begin{array}{l}
\beta_{m} H_{m} \\
L_{m}
\end{array}\right] P_{m}(k) F_{0}^{T} \\
& -F_{1} P_{m}(k-1)\left[\beta_{m} H_{m}^{T} L_{m}^{T}\right] R_{m}^{-1}\left[\begin{array}{l}
\beta_{m} H_{m} \\
L_{m}
\end{array}\right] P_{m}(k-1) F_{1}^{T}
\end{aligned}
$$

In (11) the composite measurement covariance matrix is obtained as

$R_{m}=\left[\begin{array}{cc}I & 0 \\ 0 & -\gamma^{2} I\end{array}\right]+\left[\begin{array}{l}\beta_{m} H_{m} \\ L_{m}\end{array}\right] P_{m}(k)\left[\beta_{m} H_{m}^{T} L_{m}^{T}\right](12)$

In (12), $\gamma$ is the factor that specifies the bound on the energy (variance) gain from the input energies due to the disturbances (w(.), and $\mathrm{v}()$.$) , and the input error in$ the state initial condition to the output state error energy. The HIPF filter gain is obtained as

$K_{m}=\beta_{m} P_{m}(k+1) H_{m}^{T}\left(\beta_{m}^{2} H_{m} P(k+1) H_{m}^{T}+I\right)^{-1}(13)$

Then, the measurementupdate of the state is given as $\hat{x}_{m}(k+1)=\tilde{x}_{m}(k)+K_{m}\left(z_{m}(k+1)-\beta_{m} H_{m} \tilde{x}(k)\right)$ In (14), the previous state estimate is obtained as $\tilde{x}_{m}(k)=F_{0} \hat{x}_{m}(k)+F_{1} \hat{x}_{m}(k-1)(15)$

The HIPF as such obtains the individual state and covariance estimates for each sensor, hence it requires to obtain the fused state and fused covariance estimates, and this is obtained by the state vector fusion formulae.The fused estimate of the states is given as

$\hat{x}^{f}(k+1)=\hat{x}_{1}(k+1)+\hat{P}_{1}(k+1)\left[\hat{P}_{1}(k+1)+\hat{P}_{2}(k+1)\right]^{-1}\left\{\hat{x}_{2}(k+1)-\hat{x}_{1}(k+1)\right\}$ (16)

The fused estimate of the SEG matrix is given as 


$$
\hat{P}^{f}(k+1)=\hat{P}_{1}(k+1)-\hat{P}_{1}(k+1)\left[\hat{P}_{1}(k+1)+\hat{P}_{2}(k+1)\right]^{-1} \hat{P}_{1}^{\top}(k+1)
$$

As can be seen from (16), and (17), that the fusion formulae utilize the individual estimates from the two sensors' estimates obtained in (11) and (14).

\subsection{Modified H-infinity global filtering-cum-fusion algorithm (HIGF)}

This is a decentralized algorithm and is also based on H-infinity concept [13], and has the central fusion equations. The modified form suitable to equations (1) and (2) is given next.The local filters are given for each senor as:

$$
\begin{aligned}
& \tilde{x}_{m}(k+1)=F_{0} \hat{x}_{m}(k)+F_{1} \hat{x}_{m}(k-1) \\
& \tilde{P}_{m}(k+1)=F_{0} \hat{P}_{m}(k) F_{0}^{T}+F_{1} \hat{P}_{m} F_{1}^{T}+G Q G^{T}
\end{aligned}
$$

The SEG update is given as

$\hat{P}_{m}^{-1}(k+1)=\tilde{P}_{m}^{-1}(k+1)+\left[\begin{array}{ll}\beta_{m} H_{m}^{T} & L_{m}^{T}\end{array}\right]\left[\begin{array}{lc}I & 0 \\ 0 & -\gamma^{2} I\end{array}\right]\left[\begin{array}{l}\beta_{m} H_{m} \\ L_{m}\end{array}\right]$

The local filter gains are given as

$A_{m}=I+\frac{1}{\gamma^{2}} \hat{P}_{m}(k+1) L_{m}^{T} L_{m} ; K_{m}=\beta_{m} A_{m}^{-1} \hat{P}_{m}(k+1) H_{m}^{T}$

$\hat{x}_{m}(k+1)=\tilde{x}_{m}(k)+K_{m}\left(z_{m}(k+1)-\beta_{m} H_{m} \tilde{x}(k+1)\right)$

The time propagation of the fused states and the SEG matrix is given as

$$
\begin{aligned}
& \tilde{x}^{f}(k+1)=F_{0} \hat{x}^{f}(k)+F_{1} \hat{x}^{f}(k-1) \\
& \tilde{P}^{f}(k+1)=F_{0} \hat{P}^{f}(k) F_{0}^{T}+F_{1} \hat{P}^{f}(k-1) F_{1}^{T}+G Q G^{T}
\end{aligned}
$$

The measurements update of the fusion states and covariance is given as

$$
\left(\hat{P}^{f}(k+1)\right)^{-1}=\left(\tilde{P}^{f}(k+1)\right)^{-1}+\sum_{i=1}^{m}\left\{\hat{P}_{i}^{-1}(k+1)-\tilde{P}_{i}^{-1}(k+1)\right\}+\frac{m-1}{\gamma^{2}} L^{T} L
$$

The global gain is obtained as

$$
A^{f}=I+\frac{1}{\gamma^{2}} \hat{P}^{f}(k+1) L^{T} L
$$

The global/measurement update fused state is given as

$$
\begin{aligned}
\hat{x}^{f}(k+1)= & {\left[I-\left(A^{f}\right)^{-1} \hat{P}^{f}(k+1) \beta_{m}^{2} H^{T} H_{f}\right] \tilde{x}^{f}(k+1) } \\
& +\left(A^{f}\right)^{-1} \hat{P}^{f}(k+1) \sum_{i=1}^{m}\left\{\hat{P}_{i}^{-1}(k+1) A_{i} \hat{x}_{i}(k+1)-\left[\hat{P}_{i}^{-1}(k+1) A_{i}+\beta_{m}^{2} H_{i}^{T} H \int \widetilde{x}_{i}(k)\right\}\right.
\end{aligned}
$$

In (26) the last term is given as

$$
\breve{x}_{i}(k)=F_{0} \hat{x}_{i}(k)+F_{1} \hat{x}_{i}(k-1)
$$

For this HIGF algorithm, the state vector fusion formulae (as needed in the case of HIPF are not required. Also, such explicit state vector fusion formulae are not required in case of the GFA.

\subsection{Modified H-infinity a posteriori risk sensitive filteringalgorithm (HIRF)}

This risk sensitive filter is also based on $\mathrm{H}$ infinity concept [13]; this feature is dependent on a real parameter which determines, whether more or less weightage should be given to higher or smaller errors in the estimation. The basic filter is modified to suit the equations (1) and (2).The SEG matrix propagation is obtained for each sensor as $P_{m}(k+1)=F_{0} P_{m}(k) F_{0}^{T}+F_{1} P_{m}(k-1) F_{1}^{T}+$

$$
\begin{aligned}
G Q G^{T}-F_{0} P_{m}(k)\left[\begin{array}{ll}
\beta_{m} H_{m}^{T} & L_{m}^{T}
\end{array}\right] R_{m}^{-1}\left[\begin{array}{l}
\beta_{m} H_{m} \\
L_{m}
\end{array}\right] P_{m}(k) F_{0}^{T} \\
-F_{1} P_{m}(k-1)\left[\begin{array}{ll}
\beta_{m} H_{m}^{T} & L_{m}^{T}
\end{array}\right] R_{m}^{-1}\left[\begin{array}{l}
\beta_{m} H_{m} \\
L_{m}
\end{array}\right] P_{m}(k-1) F_{1}^{T}
\end{aligned}
$$

In (28) the composite measurement covariance matrix is obtained as

$R_{m}=\left[\begin{array}{ll}R_{i} & 0 \\ 0 & \alpha^{-1} I\end{array}\right]+\left[\begin{array}{l}\beta_{m} H_{m} \\ L_{m}\end{array}\right] P_{m}(k)\left[\beta_{m} H_{m}^{T} L_{m}^{T}\right]$

In (29), $\alpha$ is a risk sensitive parameter [13].The HI filter gain is obtained as

$$
K_{m}=\beta_{m} P_{m}(k+1) H_{m}^{T}\left(\beta_{m}^{2} H_{m} P(k+1) H_{m}^{T}+I\right)^{-1} \text { (30) }
$$

Then, the measurementupdate of the state is given as $\hat{x}_{m}(k+1)=\tilde{x}_{m}(k)+K_{m}\left(z_{m}(k+1)-\beta_{m} H_{m} \tilde{x}(k)\right)(31)$ In (31), the previous state estimate is obtained as $\tilde{x}_{m}(k)=F_{0} \hat{x}_{m}(k)+F_{1} \hat{x}_{m}(k-1)(32)$

The HIRF also obtains the individual state and covariance estimates for each sensor, hence it required to obtain the fused state and fused covariance estimates, and this is given by the state vector fusion formulae (16), and (17).

\section{NONLINEAR OBSERVER BASED ON CONTINUOUS TIME DATA FUSION \\ FILTER}

Next, a continuous time data fusion filter [14] is studied and a nonlinear observer is proposed that incorporates randomly missing measurements. Let the nonlinear state model be given as (considered for say one local senor only):

$\dot{x}(t)=f(x(t), u(t))$

$y(t)=\beta H x(t)$

In (33), often control input is ignored. The scalar quantity $\beta$ is a Bernoulli sequence; in which case we could have written $\mathrm{y}(\mathrm{k})=\beta(\mathrm{k}) \mathrm{H}(\mathrm{k}) \mathrm{x}(\mathrm{k})$, without loss of any generality. A nonlinear observer for the system of (33) can be given as 
$\dot{\hat{x}}(t)=f(\hat{x}(t), u(t))+L(t)(y(t)-\hat{y}(t))$

$\hat{y}(t)=\beta H \hat{x}(t)$

In (34), L(t) is an observer gain matrix of appropriate dimension, and is given as

$$
L(t)=P(t) H^{T} R^{-1}
$$

The matrix $\mathrm{P}(\mathrm{t})$ is obtained as the solution of the observer Riccati type differential (ORD) equation which is based on the continuous time data fusion filter [14]:

$\frac{d P^{-1}(t)}{d t}=-P^{-1}(t) A(t)-A^{T}(t) P^{-1}(t)+\beta^{2} H^{T} R^{-1} H-P^{-1}(y) Q P^{-1}(t)$

One can use $Y(t)=P^{-1}(t)$ to obtain the equivalent equation which is called matrix Riccati differential equation in the literature [1]:

$\dot{Y}(t)=-Y(t) A(t)-A^{T}(t) Y(t)+\beta^{2} H^{T} R^{-1} H-Y(t) Q Y(t)$

It is seen that the observer gain is obtained from the continuous time gain fusion algorithm [14]. The required Jacobian for (37) is obtained as

$$
A(t)=\frac{\partial f(.)}{\partial \hat{x}(t)}(38)
$$

By subtracting (34) from (33), the following observer error dynamics are obtained

$$
\begin{aligned}
\dot{e}(t) & =A(t) e(t)-L(t) H \beta(x(t)-\hat{x}(t))+\phi(.) \\
& =A(t) e(t)-\beta L(t) H e(t)+\phi(.)
\end{aligned}
$$

In (39), new nonlinear function is

$$
\phi(.)=-A(t) e(t)+f(x, t)-f(\hat{x}, t)
$$

The form of (40) is obtained by adding and subtracting the terms related to the Jacobian in the original equations of the error dynamics. In (40), the short forms for $\mathrm{f}$ and $\phi$ (.) can be used for simplicity

$$
\phi(.)=\phi(x(t), \hat{x}(t), t), f(x, t)=f(x(t), t)(41)
$$

It is noted that the state errors are given as

$e(t)=x(t)-\hat{x}(t)$

3.1. Asymptotic stability of the nonlinear observer error dynamics

It is necessary to consider the following conditions $[15,16]$ for the study of the local asymptotic behaviour of the observer error dynamics of (39):

1. The solution of the matrix RTD equation (36) should be bounded

$$
p_{l} I \leq P(t) \leq p_{u} I
$$

In (43), $p_{l}, p_{u}>0$; are constants (since $\mathrm{P}(\mathrm{t})$ is theoretically, positive definite and symmetrical matrix), and are the lower and upper bounds respectively.
2. The nonlinearity (41) of the error dynamics is bounded

$$
\|\phi(.)\| \leq \rho_{1}\|x(t)-\hat{x}(t)\|^{2}(44)
$$

In (44), the bounding constant is taken greater than zero.Then, the nonlinear observer error dynamics (39) are locally asymptotically stable 35 if basically the conditions 1 and 2 are satisfied. First, the normalized LE functional is considered to establish the asymptotic stability of the error dynamics (39)as

$V(t)=e^{T}(t) Y(t) e(t)$

In $(45), Y(t)$ is the normalizing matrix and is recognized as an information matrix, and is given as $\mathrm{Y}(\mathrm{t})=\mathrm{P}^{-1}(\mathrm{t})$. The matrix $\mathrm{P}($.$) is called Gramian matrix,$ and $\mathrm{Y}($.$) the 'information matrix', or theinformation$ Gramian. In such cases, because the deterministic observers are considered, the variables $\mathrm{x}($.$) , and \mathrm{y}($.) are called as the generalized 'random' variables [12]. It is seen that the LE functional is positive definite because it is governed by the condition 1 , theinequality of (43):

$$
\frac{1}{p_{u}}\|e(t)\|^{2} \leq e^{T}(t) Y(t) e(t) \leq \frac{1}{p_{l}}\|e(t)\|^{2}
$$

The time derivative of the LE functional, (45), under the constraints governed by error dynamics (39), and (35) and (37), should be negative definite. This time derivative is obtained, after substituting these constraints and simplifying (without any approximations, and keeping the track of matrix/vector algebra consistency) as $\dot{V}(t)=-e^{T}(t) Y(t) Q Y(t) e(t)+\beta^{2} e^{T}(t) H^{T} R^{-1} H e(t)$

$$
-\beta e^{T}(t) H^{T} L^{T} Y e(t)-\beta e^{T}(t) Y(t) L H e(t)+2 e^{T}(t) Y(t) \phi(.) .
$$

The last term is so written, because the matrix $\mathrm{Y}($.$) is$ symmetrical. Now, for (47), it is assumed that $\left\|R^{-1}\right\| \leq 1 / r ;\left\|H^{T} H\right\| \leq h^{2}$, (r and $\mathrm{h}$ being positive constants) and $\|e(t)\|^{2} \leq \varepsilon^{2}$; and since, these are known and pre-specified quantities, or should be finite; one obtains, using the inequality from (44), and the observer gain from (35), in terms of respective norms:

$$
\dot{V}(t) \leq-\left\{\frac{q_{l}}{p_{u}^{2}}-\frac{2 \rho_{1}}{p_{u}}\|e(t)\|\right\}\|e(t)\|^{2}-\frac{(2-\beta) \beta h^{2}}{r}\|e(t)\|^{2}
$$

In (48), $q_{l}$ is the smallest (positive) eigenvalue of the (4) $\|e(t)\| \leq \varepsilon=\mathrm{d}$, the following condition from (48) results 
$\dot{V}(t) \leq-\left\{\frac{q_{l}}{p_{u}^{2}}-\frac{2 \rho_{1}}{p_{u}} d\right\}\|e(t)\|^{2}-\left[\frac{(2-\beta) \beta h^{2}}{r}\right]\|e(t)\|^{2}$

$$
\dot{V}(t) \leq-\left\{d<\frac{q_{l}}{2 \rho_{1} p_{u}} ; \beta<2\right\}\|e(t)\|^{2}
$$

Since, various constants and bounds (defined earlier) appearing in the $\{. ;$.$\} of (49) are positive, then for the$ specified conditions on ' $\mathrm{d}$ ' and $\beta$ in (50), it is seen that the time derivative of the Lyapunov energy functional is locally negative definite as in (50). Hence, the error dynamics of the nonlinear observer for system with randomly missing measurementsare locally asymptotically stable. This result establishes that the continuous time global data fusion filtering algorithm [14] that receives the estimates from the local filters, would also be asymptotically stable; because the asymptotic convergence result is based on the observer error dynamics that use the gain and the covariance matrix from the local continuous time data fusion filter. This is novel interpretation in this paper.

\section{PERFORMANCE EVALUATION OF THE FOUR MODIFIED ALGORITHMS}

These algorithms are implemented in MATLAB and validated using simulated data. The target tracking dynamic system model considered is given as

$$
x(k+1)=F(x)+G w(k)
$$

The system matrices are

$$
F=\left[\begin{array}{ll}
1 & T \\
0 & 1
\end{array}\right] ; G=\left[\begin{array}{c}
T^{2} / 2 \\
T
\end{array}\right]
$$

The measurement model for each sensor is given as

$z_{m}(k)=H_{m} x(k)+v_{m}(k)$

For simplicity, the state delay is not considered in MATLAB implementation; however, it can be easily incorporated. The samping interal is $0.5 \mathrm{sec}$; and additive random noise processes are considered in state and measurements. Two sensor situation is considered with dissimilar measurement noise variances. The initial conditions for the states are $x(0)=\left[\begin{array}{ll}200 & 0.5\end{array}\right]$. The state $x 1($.$) is a position and the$ state $\mathrm{x} 2($.$) is a velocity; and position state is taken as$ observations. The measurement fit error and state vector error $(\%)$ performance metrics are computed as: $\operatorname{PFE}(\mathrm{fe})=100 * \operatorname{cov}($ measurements' error $) / \operatorname{cov}($ true or actual measurements). If covariance measure is found to be ill-conditioned, then 'norm' can be used instead. Also, the H-infinity norm is evalauted as

$$
H(\text { nam })=\frac{\sum_{k=0}^{N}\left(\hat{x}^{f}(k)-x(k)^{T}\left(\hat{x}^{f}(k)-x(k)\right)\right.}{\left(\hat{x}_{0}^{f}-x\right)^{T} P_{0}^{f}\left(\hat{x}_{0}^{f}-x\right)+\sum_{k=0}^{N} w^{f}(k) u(k)+\sum_{i}^{m} \sum_{k=0}^{N} v_{i}^{T}(k) v_{i}(k)}
$$

The HI norm is the ratio of the output error (state estimate error) energy to the total input energies of the disturbances; this includes the error in the state initial conditionand all the noise variances.

The performance evaluation of the three filters is carried out for no measurement-data loss, and for randomly missing measurements (at the level of 0.95), for each sensor in turn. The sensors are differentiated here in terms of their different measurement variances; R1, and R2. The performance metrics are given in Table 1; from where it can be seen that the GFA algorithm performs better (the metrics' values marked in bold) than the three HI based filters; in both the situations, without and with measurement loss. This might be due to the fact that there is information feedback in GFA from the global to the local filters. The performance of the HIPF, HIRF (appropriate choice of the risk factor is required) and HIGF filtering/fusion algorithms is nearly similar in most cases, as these are based on the same theory of $\mathrm{H}$-infinity. As a typical case, the Figures 1-4 show the filter performance plots for the case of HIGF when there is random data loss in sensor 1; which shows satisfactory tracking. Also, it can be seen that the fusion performance of all the four algorithms, is as per the accepted inferences (from the literature on data fusion), and is very satisfactory; as also can be seen in Figure 3.

\section{CONCLUSION}

The suitable modifications in four filteringcum-fusion algorithms have been presented to incorporate the system state delay in the dynamic model, and randomly missing data in the measurement equation. The performance has been 
evaluated using MATLAB based implementations when there is measurement data loss in each sensor (in turn) and when there is no data loss in either sensor. It has been found that the gain fusion GFA filtering-cum-fusion algorithm performs better than the other three HI based algorithms; however, the latter three also exhibit consistent and good performance within themselves in the two cases of the data loss studied. Anonlinear observer, based on the continuous time filtering-cum-fusion algorithm has been presented for the system with randomly missing measurements, and asymptotic convergence result for the error dynamics has been derived based on
Lyapunov energy functional. This latter result also signifies the fact that, if the local filters are asymptotically stable, then the global filter that is an optimal combination of these local filters would also be asymptotically stable. This kind of crossutilization of the observer theoretic analysis in predicting the stability of the global data fusion algorithm is a novel interpretation in this paper. Such studies have utilization in applications of the observers and the data fusion algorithms in communications systems, wireless sensor networks, mechanical/aerospaceengineering (target tracking), and robotics.

Table 1. Performance metrics for the three modified filters

\begin{tabular}{|c|c|c|c|c|c|c|c|c|c|c|c|c|}
\hline Cond. & \multicolumn{3}{|c|}{ GFA } & \multicolumn{3}{|c|}{ HIPF } & \multicolumn{3}{|c|}{ HIRF } & \multicolumn{3}{|c|}{ HIGF } \\
\hline \multirow{6}{*}{$\begin{array}{l}\text { No } \\
\text { loss } \\
\text { of data }\end{array}$} & Hnorm & Pfe S1 & Pfe S2 & Hnorm & Pfe S1 & Pfe S2 & Hnorm & Pfe S1 & Pfe S2 & Hnorm & Pfe S1 & Pfe S2 \\
\hline & 0.004 & 0.0801 & 0.1123 & 0.0051 & 0.0815 & 0.1151 & 0.0058 & 0.0821 & 0.1151 & 0.0146 & 0.081 & 0.1148 \\
\hline & $\begin{array}{l}\text { SVE } \\
\text { in }\end{array}$ & Pos & Vel & $\begin{array}{l}\text { SVE } \\
\text { in }\end{array}$ & Pos & Vel & $\begin{array}{l}\text { SVE } \\
\text { in }\end{array}$ & Pos & Vel & $\begin{array}{l}\text { SVE } \\
\text { in }\end{array}$ & Pos & Vel \\
\hline & S1 & 0.0569 & 3.746 & S1 & 0.078 & 6.241 & S1 & 0.0798 & 7.600 & $\mathrm{~S} 1$ & 0.0757 & 4.176 \\
\hline & S2 & 0.0579 & 3.666 & $\mathrm{~S} 2$ & 0.0916 & 6.463 & S2 & 0.0909 & 6.2594 & S2 & 0.0838 & 4.467 \\
\hline & $\mathrm{F}$ & 0.0565 & 3.65 & $\mathrm{~F}$ & 0.0649 & 5.942 & $\mathrm{~F}$ & 0.0687 & 6.5977 & $\mathrm{~F}$ & 0.071 & 6.658 \\
\hline \multirow{6}{*}{$\begin{array}{l}\text { Data } \\
\text { loss in } \\
\text { Sensor } \\
1: S 1 ; \\
\text { F- } \\
\text { fused }\end{array}$} & Hnorm & Pfe S1 & Pfe S2 & Hnorm & Pfe S1 & Pfe S2 & Hnorm & Pfe S1 & Pfe S2 & Hnorm & Pfe S1 & Pfe S2 \\
\hline & 0.0041 & 0.0264 & 0.1123 & 0.0051 & 0.0271 & 0.1151 & 0.0058 & 0.0273 & 0.1151 & 0.0713 & 0.027 & 0.1148 \\
\hline & $\begin{array}{l}\text { SVE } \\
\text { in }\end{array}$ & Pos & Vel & $\begin{array}{l}\text { SVE } \\
\text { in }\end{array}$ & Pos & Vel & $\begin{array}{l}\text { SVE } \\
\text { in }\end{array}$ & Pos & Vel & $\begin{array}{l}\text { SVE } \\
\text { in }\end{array}$ & Pos & Vel \\
\hline & S1 & 0.0577 & 3.7197 & $\mathrm{~S} 1$ & 0.0814 & 5.96 & S1 & 0.0817 & 7.299 & $\mathrm{~S} 1$ & 0.0801 & 4.2782 \\
\hline & S2 & 0.0588 & 3.6391 & $\mathrm{~S} 2$ & 0.0916 & 6.4625 & $\mathrm{~S} 2$ & 0.0909 & 6.2594 & S2 & 0.0838 & 4.487 \\
\hline & $\mathrm{F}$ & 0.0573 & 3.6235 & $\mathrm{~F}$ & 0.0654 & 5.7935 & $\mathrm{~F}$ & 0.0690 & 5.4209 & $\mathrm{~F}$ & 0.1568 & 6.6116 \\
\hline \multirow{6}{*}{$\begin{array}{l}\text { Data } \\
\text { loss in } \\
\text { Sensor } \\
2: S 2 ; \\
\text { F- } \\
\text { fused }\end{array}$} & Hnorm & Pfe S1 & Pfe S2 & Hnorm & Pfe S1 & Pfe S2 & Hnorm & Pfe S1 & Pfe S2 & Hnorm & Pfe S1 & Pfe S2 \\
\hline & 0.0041 & 0.08 & 0.037 & 0.0051 & 0.0815 & 0.0378 & 0.0058 & 0.0821 & 0.0378 & 0.0709 & 0.0810 & 0.0378 \\
\hline & $\begin{array}{l}\text { SVE } \\
\text { in }\end{array}$ & Pos & Vel & $\begin{array}{l}\text { SVE } \\
\text { in }\end{array}$ & Pos & Vel & $\begin{array}{l}\text { SVE } \\
\text { in }\end{array}$ & Pos & Vel & $\begin{array}{l}\text { SVE } \\
\text { in }\end{array}$ & Pos & Vel \\
\hline & S1 & 0.0576 & 3.724 & S1 & 0.0779 & 6.241 & S1 & 0.0798 & 7.6 & S1 & 0.0757 & 4.176 \\
\hline & S2 & 0.0588 & 3.6553 & S2 & 0.096 & 6.2277 & S2 & 0.0958 & 6.1444 & S2 & 0.0901 & 4.637 \\
\hline & $\mathrm{F}$ & 0.0572 & 3.6342 & $\mathrm{~F}$ & 0.0648 & 5.7434 & $\mathrm{~F}$ & 0.0690 & 6.4876 & $\mathrm{~F}$ & 0.1563 & 6.5616 \\
\hline
\end{tabular}

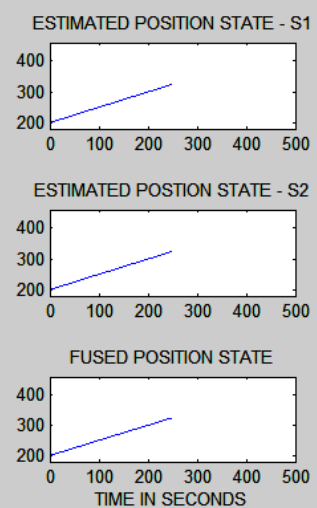

TIME IN SECONDS

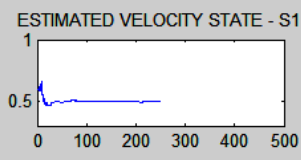

ESTIMATED VELOCITY STATE - S2

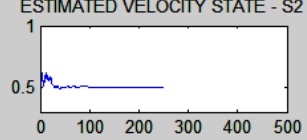

FUSED VELOCITY STATE

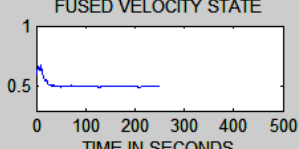

TIME IN SECONDS

Figure 1 Time histories of states (HIGF)
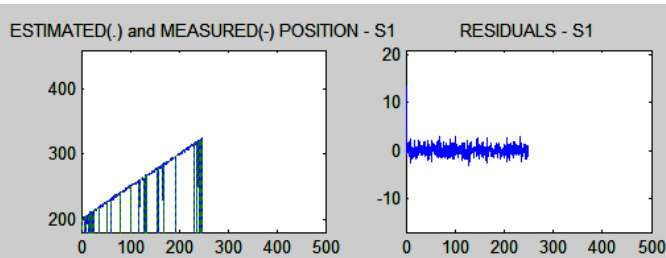

ESTIMATED(.) and MEASURED(-) POSITION - S2
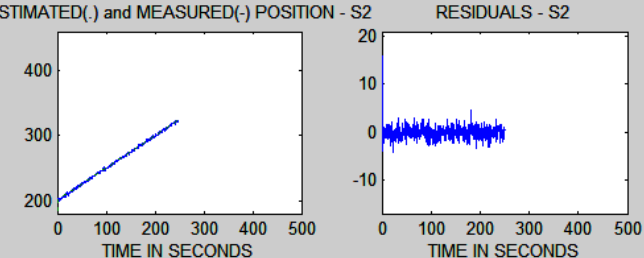

Figure 2 Measurements and residuals (HIGF) 


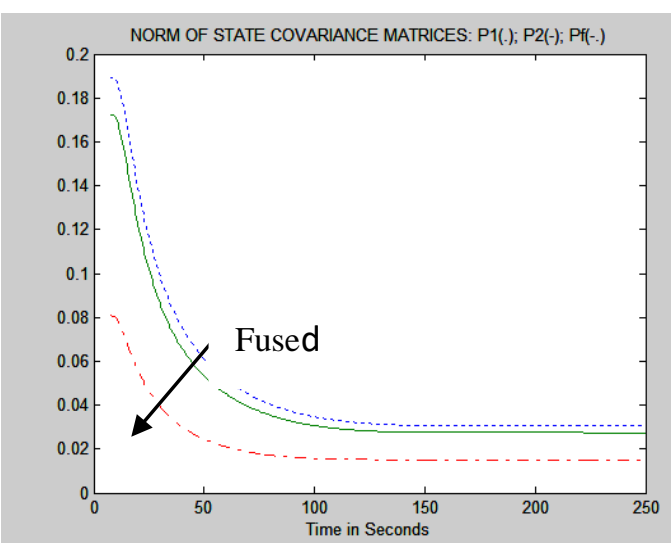

Figure 3 Norms of $\mathrm{P}($.$) ; individual and fused$

\section{REFERENCES}

[1]. Raol, J. R. Data Fusion Mathematics-Theory and Practice, CRC Press, Taylor \& Francis, Florida, USA, July 2015.

[2]. Sinopoli, Bruno, et al. Kalman filtering with intermittent observations. IEEE Transaction on Automatic Control, Vol. 49, No. 9, pp. 1453-1464, 2004.

[3]. Nelwamondo, Fulufhelo Vincent, and Tshilidzi Marwala. Techniques for handling missing data: applications to online condition monitoring. Int. Jl. of Innovative Comput. Inf. Control Vol. 4, No. 6, pp. 1507-1526, 2008.

[4]. Yang, Rongni, Peng Shi, and Guo-Ping Liu. Filtering for discrete-time networked nonlinear systems with mixed random delays and packet dropouts. IEEE Transaction on Automatic Control, Vol. 56, No. 11, pp. 2655-2660, 2011.

[5]. Chen, D., and Xu Long. Optimal Kalman filtering for a class of state delay systems with randomly multiple sensor delays. Abstract and Applied Analysis. Hindawi Publishing Corporation. Vol. 2014, ID716716, pp. 1-10. 2014.

[6]. Cipra, T., and Romera, R. Kalman filter with outliers and missing observations. Sociedad de Estadistica e Investigacion Operativa. Test Vol. 6, No. 2, pp. 379-395, 1997.

[7]. Savkin, A. V., Petersen I. R., and Reza Moheimani, S. O. Model validation and state estimation for uncertain continuous-time systems with missing discrete-continuous data. Computers and electrical engineering, Pergamon, Vol. 25, pp. 29-43, 1999.

[8]. Tresp, V., and Hofmann, R. Nonlinear timeseries prediction with missing and noisy data. Neural Computation, Vol. 10, pp. 731747, 1998.

[9]. Basin, M.V., Rodriguez-Gonzalez, J., and Martinez-Zuniga, R. Optimal filtering for
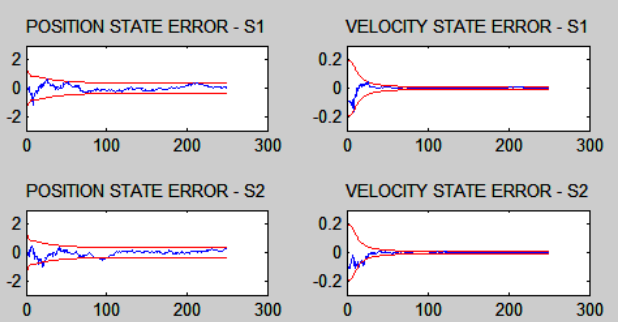

POSITION STATE ERROR - FUSED


Figure 4 The state errors with their bounds.

linear systems with state delay. International Jl. of Pure and Applied Mathematics. Vol. 10, No. 3, pp. 241-255, 2004. 